University of Wollongong

Research Online

Faculty of Engineering and Information

Faculty of Engineering and Information

Sciences - Papers: Part A

Sciences

$1-1-2015$

\title{
An analysis of a standard reactor cascade and a step-feed reactor cascade for biological processes described by monod kinetics
}

Harvinder S. Sidhu

University of New South Wales, h.sidhu@adfa.edu.au

Mark lan Nelson

University of Wollongong, mnelson@uow.edu.au

Easwaran Balakrishnan

Sultan Qaboos University, balak@squ.edu.pm

Follow this and additional works at: https://ro.uow.edu.au/eispapers

Part of the Engineering Commons, and the Science and Technology Studies Commons

Research Online is the open access institutional repository for the University of Wollongong. For further information contact the UOW Library: research-pubs@uow.edu.au 


\title{
An analysis of a standard reactor cascade and a step-feed reactor cascade for biological processes described by monod kinetics
}

\author{
Abstract \\ We analyse the steady-state operation of two types of reactor cascade without recycle. The first is a \\ standard reactor cascade in which the feed stream enters into the first reactor. The second is a step-feed \\ reactor cascade in which an equal proportion of the feed stream enters each reactor in the cascade. The \\ reaction is assumed to be a biological process governed by Monod growth kinetics with a decay \\ coefficient for the microorganisms. The steady-states of both models are found for an arbitrary number of \\ reactors and their stability determined as a function of the residence time. We show that in a step-feed \\ reactor cascade the substrate and biomass concentrations leaving the reactor of the cascade are \\ identical to those leaving the first reactor of the cascade. We further show that this result is true for a \\ general specific growth rate of the form $\mu(S, X)$. Thus for such processes the non-standard cascade \\ offers no advantage over that of a single reactor. This is surprising because the use of a non-standard \\ cascade has been proposed as a mechanism to improve the biological treatment of wastewater.

\section{Disciplines} \\ Engineering | Science and Technology Studies

\section{Publication Details} \\ Sidhu, H. S., Nelson, M. I. \& Balakrishnan, E. (2015). An analysis of a standard reactor cascade and a step- \\ feed reactor cascade for biological processes described by monod kinetics. Chemical Product and \\ Process Modeling, 10 (1), 27-37.
}


Harvinder S. Sidhu, Mark Ian Nelson* and Easwaran Balakrishnan

\section{An Analysis of a Standard Reactor Cascade and a Step-Feed Reactor Cascade for Biological Processes Described by Monod Kinetics}

\begin{abstract}
We analyse the steady-state operation of two types of reactor cascade without recycle. The first is a standard reactor cascade in which the feed stream enters into the first reactor. The second is a step-feed reactor cascade in which an equal proportion of the feed stream enters each reactor in the cascade. The reaction is assumed to be a biological process governed by Monod growth kinetics with a decay coefficient for the microorganisms. The steady-states of both models are found for an arbitrary number of reactors and their stability determined as a function of the residence time. We show that in a step-feed reactor cascade the substrate and biomass concentrations leaving the reactor of the cascade are identical to those leaving the first reactor of the cascade. We further show that this result is true for a general specific growth rate of the form $\mu(S, X)$. Thus for such processes the non-standard cascade offers no advantage over that of a single reactor. This is surprising because the use of a non-standard cascade has been proposed as a mechanism to improve the biological treatment of wastewater.
\end{abstract}

Keywords: multistage continuous culture, step-feed reactor, modelling

DOI 10.1515/cppm-2014-0022

\section{Introduction}

A continuous flow bioreactor is a well-stirred vessel containing microorganisms $(X)$ through which a substrate $(S)$

\footnotetext{
*Corresponding author: Mark Ian Nelson, School of Mathematics and Applied Statistics, University of Wollongong, Northfields Avenue, Wollongong, NSW 2522, Australia, E-mail: mnelson@uow.edu.au http://orcid.org/0000-0002-3964-5928

Harvinder S. Sidhu, School of Physical, Environmental and Mathematical Sciences, UNSW at ADFA, Canberra, ACT, Australia, E-mail: h.sidhu@adfa.edu.au

Easwaran Balakrishnan, Department of Mathematics and Statistics, Sultan Qaboos University, Al Khoudh, Muscat 123, Oman, E-mail: balak@squ.edu.om
}

flows at a continuous rate. The microorganisms grow in the vessel through consumption of the substrate to produce more microorganisms and a product $(P)$. Unused substrate, microorganisms, and the product flow out of the reactor.

The performance of a chemical process can often be improved by replacing a single continuous flow bioreactor with a sequence of bioreactors. In a standard reactorcascade, the feed stream flows into the first reactor of the cascade. Thereafter the product stream from the $i$ th reactor of the cascade acts as the feed stream for the $(i+1) \mathrm{h}$ reactor of the cascade. This process is illustrated in Figure 1. We refer to the configuration shown in this figure as the "standard reactor cascade" (SRC). These cascades are particularly attractive in the processing of wastewaters due to the low effluent concentrations which can be attained.

An alternative reactor configuration is to use a stepfeed design $[1,2]$. This is similar to the standard configuration in that the product stream from the ith reactor flows into the $(i+1)$ th reactor. It differs in how the feed stream is treated. Instead of all the feed stream entering the first reactor, a bypass is used so that an equal proportion of the feed stream enters into each reactor of the cascade. This configuration is illustrated in Figure 2. We refer to this configuration as a "step-feed reactor cascade" (SFRC). This reactor design is common in the implementation of the activated sludge process $[1,2]$. In a more general version of this design, sometimes called a multistream reactor cascade, the reactors in the cascade do not necessarily receive an equal proportion of the feed stream. We do not consider such reactor designs.

We analyse the steady-state behaviour of a biological process in both the standard reactor cascade and the nonstandard reactor cascade. The main novelty in this work is our analysis of the non-standard reactor cascade. We model the degradation of a biodegradable organic material using the Monod expression [3]. This simple approach is widely used to model biochemical growth processes.

We are primarily interested in applications associated with the biological treatment of wastewaters. Thus the primary variable of interest is the effluent 


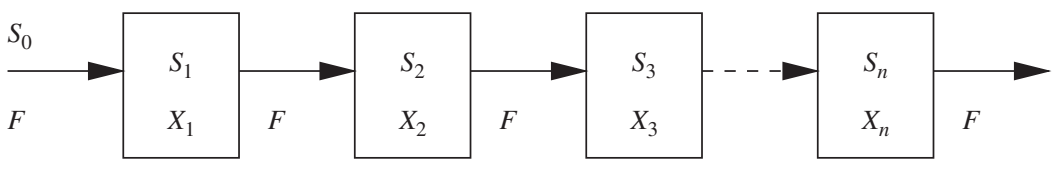

Figure 1: Schematic figure of a "standard" reactor cascade with $n$ reactors.

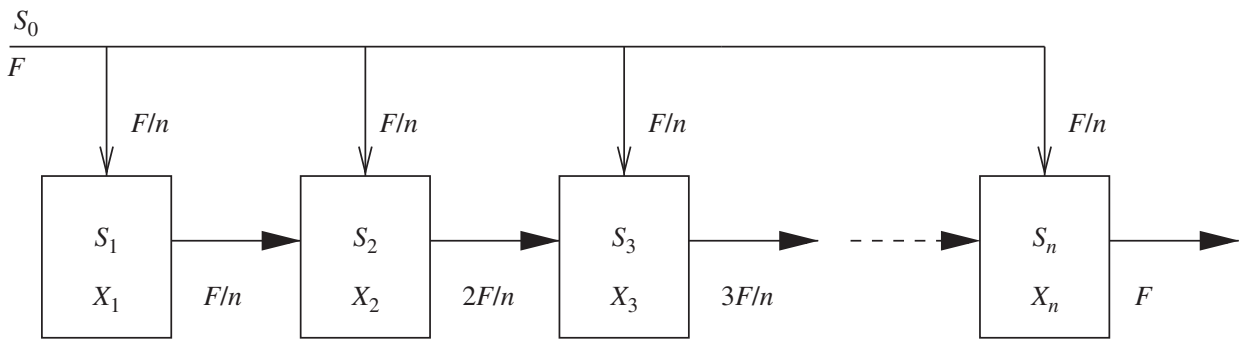

Figure 2: Schematic figure of a step-feed reactor cascade consisting of $n$ reactors.

concentration leaving the cascade. We will use this to gauge the performance of the two cascade systems.

There is a long history of mathematical work analysing flow reactor models with applications in bioprocess engineering. A short overview of some of the historical developments in this area in a single tank, particularly regarding the use of the Monod model, is presented in Ref. [4]. The early work on modelling reactor cascades is summarised in Ref. [5]. More recent work concentrating on biological wastewater treatment is contained in Ref. [6].

There is a considerable body of literature regarding the analysis of processes carried out in reactor cascades. It is outside the scope of this paper to review this body of literature. However, we indicate some of the directions taken in recent years.

Abu-Reesh [7] investigated the optimisation of an industrial process for the degradation of phenol in a standard reactor cascade containing up to five reactors. The specific-growth rate chosen was the Haldane expression, which is often used to model processes exhibiting substrate inhibition. The objective was to minimise the volume required for a certain percentage of phenol removal. It was shown that the optimal design involved unequal reactor volumes at relatively high feed concentration and high substrate conversion. It was assumed that biomass decay does not occur.

Dramé et al. [8] analysed a standard reactor cascade model, considering two abstract representations for the specific growth rate. When the specific growth rate is a monotonically increasing function, such as the Monod expression, then there is at most one no-washout steady-state solution in each reactor. When the specific growth rate represents a generalised formulation for substrate inhibition, i.e. it initially increases on some substrate concentration range and then it decreases, then in each reactor there are at most three no-washout steadystate solutions. It was assumed that biomass decay does not occur.

The steady-state treatment of industrial wastewaters in a cascade reactor was analysed by Alqahtani et al. $(9,10)$. In the former, a settling unit placed around the exit stream of reactor $i$ recycles the effluent stream into the feed stream for reactor $i$. The latter considered the scenario in which a settling unit recycles from the final reactor in the cascade to the first. The Contois growth-rate expression was used and biomass decay was considered.

Carlsoon and Zambrano [11] compared the effluent concentration leaving a two-reactor cascade subject to either Monod or Contois kinetics. In both cases it is assumed that the biomass do not decay. Although expressions for the steady-state solutions were found, stability was not determined.

It is worth noting that in none of these papers is the step-feed reactor cascade analysed. Even for the standard reactor cascade, it is often assumed that biomass do not decay $[7,8,11]$.

The operation of processes carried out in continuous flow reactors is routinely carried out by finding the steady-states of the appropriate model and determining their stability. In the absence of a control scheme, reactors can only be operated at stable steady-state solutions. Thus knowledge of stable steady-state solutions is essential as a stepping stone to understanding any process. The main contributions of the current work are:

- we find the steady-state solutions for a standard reactor cascade;

- we determine the stability of the solutions;

- we find the steady-state solutions of a step-feed reactor cascade;

- we determine the stability of the solutions. 
- we show that the average time a particle spends in a step-feed reactor cascade is identical to the average time that a particle spends in a standard reactor cascade.

Although the standard reactor cascade has been widely investigated, stability results have only been presented previously for the case when the biomass do not decay.

Our most significant contribution is that, using the above results, we establish a very surprising result. Namely that the effluent concentration leaving a $n$-reactor step-feed reactor cascade is the same as that leaving a single-reactor. Thus, the performance of these two reactor configures is identical and there is no advantage gained from operating a step-feed reactor cascade.

\section{Equations}

In this section, we write down the model equations for the concentration of microorganisms and substrate within a "standard reactor cascade" (SRC) and a "step-feed reactor cascade" (SFRC). It is assumed that all reactors are wellstirred and well aerated. The assumption that a reactor is well-stirred means that the substrate is instantaneously and homogeneously mixed with the reactor contents. The assumption that a reactor is well aerated means that oxygen is not a rate-limiting substance. It is also assumed that the flow through each bioreactor is sufficiently fast so that cellgrowth does not occur on the reactor walls, i.e. there is no biofilm on the reactor walls, and that operating conditions such as the $\mathrm{pH}$, temperature and other environmental conditions are automatically controlled so as to remain constant.

The model equations for the SRC and SFRC models are stated in Sections 2.1 and 2.2 respectively. In Section 2.3 we summarise some useful results for the behaviour of a single reactor system.

\subsection{Standard reactor cascade (SRC)}

The dimensional and non-dimensional model equations for a standard reactor cascade are given in Sections 2.1.1 and 2.1.2 respectively.

\subsubsection{The dimensional model}

The model equations in the $i$ th reactor of a SRC containing $n$ reactors are given by $(1 \leq i \leq n)$

$$
\begin{gathered}
V_{i} \frac{d S_{i}}{d t}=F\left(S_{i-1}-S_{i}\right)-V_{i} \frac{\mu_{m} S_{i} X_{i}}{K_{s}+S_{i}} \cdot \frac{1}{\alpha}, \\
V_{i} \frac{d X_{i}}{d t}=F\left(X_{i-1}-X_{i}\right)+V_{i} \frac{\mu_{m} S_{i} X_{i}}{K_{s}+S_{i}}-V_{i} k_{d} X_{i} .
\end{gathered}
$$

The residence time in each reactor is given by

$$
\tau_{i}=\frac{V_{i}}{F} .
$$

The parameters and variables are defined in Appendix B.

We consider the case when all reactors have equal volume $\left(V_{1}=V_{2} \ldots=V\right)$. Thus the residence time in reach reactor of the cascade $\left(\tau_{i}\right)$ is related to the total residence time in the cascade $\left(\tau_{t}\right)$ by

$$
\tau_{i}=\frac{\tau_{t}}{n}
$$

For a specific wastewater, a given biological community and a particular set of environmental conditions the parameters $K_{s}, k_{d}, \alpha$ and $\mu_{m}$ are fixed. The parameters that can be varied are $S_{0}, X_{0}$ and $\tau_{i}$.

In our numerical simulations, we use typical parameter values for the activated sludge treatment of wastewater [12]. These are $K_{s}=100(\mathrm{mgCOD})(\mathrm{L})^{-1}$, $S_{0}=4000 \mathrm{mg} \mathrm{COD} \mathrm{L}^{-1}, \quad k_{d}=0.028$ day $^{-1}, \quad \alpha=0.5 \mathrm{mg}$ MLSS mg COD ${ }^{-1}$, and $\mu_{m}=1.0\left(\right.$ day $\left.^{-1}\right)$.

\subsubsection{The dimensionless model}

By introducing dimensionless variables for the substrate concentration in the $i$ th reactor of the cascade $\left[S_{i}^{*}=S_{i} / K_{s}\right]$, the microorganism concentration in the $i$ th reactor of the cascade $\left[X_{i}^{*}=X_{i} /\left(\alpha K_{s}\right)\right]$ and time $\left[t^{*}=\mu_{m} t\right]$ the dimensional model for the $i$ th reactor of the cascade can be written in the scaled form

$$
\frac{d S_{i}^{*}}{d t^{*}}=\frac{1}{\tau_{i}^{*}}\left(S_{i-1}^{*}-S_{i}^{*}\right)-\frac{S_{i}^{*} X_{i}^{*}}{1+S_{i}^{*}},
$$

$$
\frac{d X_{i}^{*}}{d t^{*}}=\frac{1}{\tau_{i}^{*}}\left(X_{i-1}^{*}-X_{i}^{*}\right)+\frac{S_{i}^{*} X_{i}^{*}}{1+S_{i}^{*}}-k_{d}^{*} X_{i}^{*},
$$

where the parameter groups are: the dimensionless substrate concentration in the feed $\left[S_{0}^{*}=S_{0} / K_{s}\right]$, the dimensionless microorganism concentration in the feed $\left[X_{0}^{*}=X_{0} /\left(\alpha K_{s}\right)\right]$, the dimensionless decay rate $\left[k_{d}^{*}=k_{d} / \mu_{m}\right]$ and the dimensionless residence time $\left[\tau_{i}^{*}=V_{i} \mu_{m} / F\right]$.

We again assume that the growth medium fed into the bioreactor is sterile, i.e. there are no microorganisms in the influent $\left(X_{0}=X_{0}^{*}=0\right)$. Furthermore, we assume that $S_{0}^{*}>0$. 
The values of the dimensionless parameters are $S_{0}^{*}=40$ and $k_{d}^{*}=0.028$.

\subsection{Step-feed reactor cascade (SFRC)}

The dimensional and non-dimensional model equations for a standard reactor cascade are given in Sections 2.2.1 and 2.2.2 respectively.

\subsubsection{The dimensional model}

The model equations in the $i$ th reactor of a SFRC contain ing $n$ reactors are $(1 \leq i \leq n)$

$$
\begin{gathered}
\frac{d S_{i}}{d t}=\frac{(i-1)}{n \tau_{i}}\left(S_{i-1}-S_{i}\right)+\frac{1}{n \tau_{i}}\left(S_{0}-S_{i}\right)-\frac{\mu_{m} S_{i} X_{i}}{K_{s}+S_{i}} \cdot \frac{1}{\alpha} \\
\frac{d X_{i}}{d t}=\frac{(i-1)}{n \tau_{i}}\left(X_{i-1}-X_{i}\right)+\frac{1}{n \tau_{i}}\left(X_{0}-X_{i}\right)+\frac{\mu_{m} S_{i} X_{i}}{K_{s}+S_{i}}-k_{d} X_{i},
\end{gathered}
$$

where the model parameters are defined in Section 2.1.1.

We consider the case when all reactors have equal volume $\left(V_{1}=V_{2} \ldots=V\right)$. We define a nominal residence time $\left(\tau_{\text {nom }}\right)$ for the SFRC based upon the feed flow rate $(F)$ and the total reactor volume in the cascade $(n V)$. This gives

$$
\tau_{\mathrm{nom}}=\frac{n V}{F}=n \tau_{i}
$$

Thus in eqs (6) and (7) we can interpret the product $n \tau_{i}$ as the nominal residence time in the SFRC.

\subsubsection{The dimensionless model}

Using the dimensionless variables introduced in Section 2.1.2 the equations for the $i$ th reactor of the SFRC are

$$
\frac{d S_{i}^{*}}{d t^{*}}=\frac{(i-1)}{n \tau_{i}^{*}}\left(S_{i-1}^{*}-S_{i}^{*}\right)+\frac{1}{n \tau_{i}^{*}}\left(S_{0}^{*}-S_{i}^{*}\right)-\frac{S_{i}^{*} X_{i}^{*}}{1+S_{i}^{*}},
$$

$\frac{d X_{i}^{*}}{d t^{*}}=\frac{(i-1)}{n \tau_{i}^{*}}\left(X_{i-1}^{*}-X_{i}^{*}\right)+\frac{1}{n \tau_{i}^{*}}\left(X_{0}^{*}-X_{i}^{*}\right)+\frac{S_{i}^{*} X_{i}^{*}}{1+S_{i}^{*}}-k_{d}^{*} X_{i}^{*}$,

where the parameter groups are defined in Section 2.1.2.

From now on we assume that the growth medium fed into the bioreactor is sterile, i.e. there are no microorganisms in the influent $\left(X_{0}=X_{0}^{*}=0\right)$. Furthermore, we assume that $S_{0}^{*}>0$.

\subsection{Washout conditions for a reactor cascade}

In this section, we provide some results on the behaviour of a single reactor system [4] and apply them to derive the washout condition for a SRC and a SFRC.

The steady-state solution in a single reactor, put $i=1$ in eqs (4) and (5) and drop the subscripts, is given by

Washout branch $\left(S^{*}, X^{*}\right)=\left(S^{*}, 0\right)$. No-washout branch

$$
\begin{aligned}
S^{*}, X^{*} & =\left(\hat{S}^{*}, \frac{S_{0}^{*}-\hat{S}^{*}}{1+k_{d}^{*} \tau^{*}}\right), \\
\hat{S}^{*} & =\frac{1+k_{d}^{*} \tau^{*}}{\left(1-k_{d}^{*}\right) \tau^{*}-1}
\end{aligned}
$$

The no-washout branch is only physically meaningful when the substrate and cell-mass concentrations are positive $\left(S^{*}>0, X^{*}>0\right)$. This happens when

$$
\begin{gathered}
\tau^{*}>\frac{S_{0}^{*}}{S_{0}^{*}-\left(1+S_{0}^{*}\right) k_{d}^{*}}, \\
0 \leq k_{d}^{*}<\frac{S_{0}^{*}}{1+S_{0}^{*}},
\end{gathered}
$$

which requires that $0 \leq k_{d}^{*}<1$. Henceforth we assume that this condition holds. The washout branch is stable when the no-washout branch is not physically meaningful. The washout branch is unstable when the no-washout branch is physically meaningful. The no-washout branch is stable when it is physically meaningful.

A transcritical bifurcation occurs when the residence time takes the value

$$
\tau_{\mathrm{tr}}^{*}=\frac{1+S_{0}^{*}}{S_{0}^{*}-k_{d}^{*}\left(1+S_{0}^{*}\right)} .
$$

At this value of the residence time the washout solution branch intersects the no-washout solution branch $\left[\left(S^{*}, X^{*}\right)=\left(S_{0}^{*}, 0\right)\right]$. For the parameter values given in Section 2.1.1, we have $\tau_{\mathrm{tr}}^{*}=1.055$, which corresponds to $\tau_{\mathrm{tr}}=1.055$ day.

Applying these results to a SRC of $n$ reactors, the condition for the transcritical bifurcation is given by 


$$
\tau_{1 . \mathrm{tr}}^{*}=n \cdot \frac{1+S_{0}^{*}}{S_{0}^{*}-k_{d}^{*}\left(1+S_{0}^{*}\right)} .
$$

Substituting $\tau^{*}=\tau_{t}^{*} / n$ in eq. (11) the effluent concentration leaving the first reactor is given by

$$
S_{1 . \mathrm{src}}^{*}=\frac{n+k_{d}^{*} \tau_{1}^{*}}{\left(1-k_{d}^{*}\right) \tau_{t}^{*}-n} .
$$

Thus the effluent concentration leaving the first reactor, for a fixed total residence time, is an increasing function of the number of reactors in the cascade.

Applying these results to a SFRC of $n$ reactors the condition for the transcritical bifurcation is given by

$$
\tau_{\mathrm{nom}, \mathrm{tr}}^{*}=\frac{1+S_{0}^{*}}{S_{0}^{*}-k_{d}^{*}\left(1+S_{0}^{*}\right)} .
$$

The effluent concentration leaving the first reactor is given by

$$
S_{1 . \mathrm{src}}^{*}=\frac{1+k_{d}^{*} \tau_{\mathrm{nom}}^{*}}{\left(1-k_{d}^{*}\right) \tau_{\mathrm{nom}}^{*}-1} .
$$

Thus the effluent concentration leaving the first reactor of a SFRC is independent of the number of reactors in the cascade and only depends upon the nominal residence time of the cascade.

\section{Results for a standard reactor cascade (SRC)}

The assumption that there is no recycling in the cascade means that the process variables in the first reactor $\left(S_{1}, X_{1}\right)$ are independent of the process variables in all other reactors and that the process variables in the $i$ th reactor $\left(S_{i}, X_{i}, i \neq 1\right)$ depend only upon those in the preceding reactor $\left(S_{i-1}, X_{i-1}\right)$. This significantly simplifies the study of system (4) and (5).

In Section 3.1 we show that, provided that washout does not occur in the first reactor, the system has a unique steady-state solution and give the steady-state values of the substrate and cell-mass concentrations in the $i$ th reactor of the cascade. Note that washout cannot occur in the later reactors if it did not occur in the first one. Washout in reactor $i$ corresponds to a steady-state solution $X_{i}^{*}=0$. However, by inspection $X_{i}^{*}=0$ is not a steady-state solution of the model if $X_{i-1}^{*} \neq 0$. In Section 3.2, the steady-state solution is shown to be locally stable. In these sections the residence times in each reactor are arbitrary.

\subsection{Steady-state solution branches}

The steady-state solutions in the ith reactor of the cascade $(i>1)$ are found by solving the algebraic equations

$$
\begin{aligned}
& f\left(S_{i}^{*}, X_{i}^{*}\right)=\frac{1}{\tau_{i}^{*}}\left(S_{i-1}^{*}-S_{i}^{*}\right)-\frac{S_{i}^{*} X_{i}^{*}}{1+S_{i}^{*}}=0, \\
& g\left(S_{i}^{*}, X_{i}^{*}\right)=\frac{1}{\tau_{i}^{*}}\left(X_{i-1}^{*}-X_{i}^{*}\right)+\frac{S_{i}^{*} X_{i}^{*}}{1+S_{i}^{*}}-k_{d}^{*} X_{i}^{*}=0 .
\end{aligned}
$$

The solution of these equations is given by

$$
\left(S_{i}^{*}, X_{i}^{*}\right)=\left(S_{i}^{*}, \frac{1}{\tau_{i}^{*}}\left(S_{i-1}^{*}-S_{i}^{*}\right) \frac{1+S_{i}^{*}}{S_{i}^{*}}\right),
$$

where the dimensionless substrate concentration $S_{i}^{*}$ satisfies the quadratic equation

$$
G\left(S_{i}^{*}\right)=a_{i} S_{i}^{*^{2}}+b_{i} S_{i}^{*}+c_{i}=0,
$$

where the coefficients are given by

$$
\begin{aligned}
& a_{i}=\left(1-k_{d}^{*}\right) \tau_{i}^{*}-1 \\
& b_{i}=-a_{i} S_{i-1}^{*}-\tau_{i}^{*} X_{i-1}^{*}-\left(1+k_{d}^{*} \tau_{i}^{*}\right) \\
& c_{i}=\left(1+k_{d}^{*} \tau_{i}^{*}\right) S_{i-1}^{*} .
\end{aligned}
$$

The solution of the quadratic equation is given by

$$
S_{i}^{*}=\frac{-b_{i} \pm \sqrt{b_{i}^{2}-4 a_{i} c_{i}}}{2 a_{i}} .
$$

We show in Appendix A that the solution corresponding to the choice of the positive square root in eq. (16) is not physically meaningful as either the substrate concentration $\left(S_{i}^{*}\right)$ or the cell mass concentration $\left(X_{i}^{*}\right)$ are negative. However, the solution corresponding to the choice of the negative square root is physically meaningful; both the cell mass and substrate concentrations are positive. Thus, given that washout does not occur in the first reactor, the solution in each reactor in the cascade is unique.

The dimensionless form of eqs (14) and (15) in the case when $k_{d}^{*}=0$ was provided by Herbert [13, eqs (28) and (29)] and, for the special case of a two-reactor cascade, by Carlsoon and Zambrano [11, eq. (12)]. In the general case, $k_{d}^{*}>0$, the dimensionless form was stated by Grady Jr and Lim [14, eqs (12.43) and (12.44)]. In none of these cases was it proved that the physically meaningful solution is always given by the choice of the negative square root sign. Similarly, in none of these cases was the stability determined. 


\subsection{Stability of the steady-state solutions}

The Jacobian matrix for a $n$-reactor cascade is given by

$$
J=\left(\begin{array}{ccccc}
J_{1} & \mathbf{0} & \mathbf{0} & \mathbf{0} & \ldots \\
B_{2} & J_{2} & \mathbf{0} & \mathbf{0} & \ldots \\
\mathbf{0} & B_{3} & J_{3} & \mathbf{0} & \ldots \\
\mathbf{0} & \ddots & \ddots & \ddots & \ddots
\end{array}\right) .
$$

This matrix has size $2 n \times 2 n$. The blocks $B_{i}$ and $J_{i}$ each have size $2 \times 2$ and are defined by

$$
\begin{gathered}
B_{i}=\left(\begin{array}{cc}
\frac{1}{\tau_{i}^{*}} & 0, \\
0 & \frac{1}{\tau_{i}^{*}}
\end{array}\right), \\
J_{i}=\left(\begin{array}{cc}
-\frac{1}{\tau_{i}^{*}}-\frac{X_{i}^{*}}{\left(1+S_{i}^{*}\right)^{2}} & -\frac{S_{i}^{*}}{1+S_{i}^{*}} \\
\frac{X_{i}^{*}}{\left(1+S_{i}^{*}\right)^{2}} & \frac{-1}{\tau_{i}^{*}}+\frac{S_{i}^{*}}{1+S_{i}^{*}}-k_{d}^{*}
\end{array}\right),
\end{gathered}
$$

and $\mathbf{0}$ represents the zero matrix of size $2 \times 2$. It follows from the structure of the Jacobian matrix that there are two eigenvalues associated with each block $J_{i}$. The block $J_{1}$ has two eigenvalues with negative real parts when (Section 2.3)

$$
\begin{gathered}
\tau^{*}>\frac{\left(1+S_{0}^{*}\right)}{S_{0}^{*}-\left(1+S_{0}^{*}\right) k_{d}^{*}}, \\
0 \leq k_{d}^{*}<\frac{S_{0}^{*}}{1+S_{0}^{*}} .
\end{gathered}
$$

The Jacobian matrix $J_{i}(i>2)$ can be re-written in the form

$$
J_{i}=\left(\begin{array}{cc}
-\frac{1}{\tau_{i}^{*}}-\frac{X_{i}^{*}}{\left(1+S_{i}^{*}\right)^{2}} & -\frac{S_{i}^{*}}{1+S_{i}^{*}} \\
\frac{X_{i}^{*}}{\left(1+S_{i}^{*}\right)^{2}} & \frac{-1}{\tau_{i}^{*}} \cdot \frac{X_{i-1}^{*}}{X_{i}^{*}}
\end{array}\right) .
$$

The simplification of the expression at position $J_{i}(2,2)$ follows from eq. (5): along the no-washout branch we have

$$
\frac{-1}{\tau_{i}^{*}}+\frac{S_{i}^{*}}{1+S_{i}^{*}}-k_{d}^{*}=-\frac{X_{i-1}^{*}}{X_{i}^{*}} \cdot \frac{1}{\tau_{i}^{*}},
$$

The eigenvalues of the $2 \times 2$ matrix $J_{i}$ have negative real parts when the determinant (det $J$ ) and trace of $J_{i}$ (trace $J$ ) are positive and negative, respectively. We have

$$
\begin{gathered}
\operatorname{det} J=\left(\frac{1}{\tau_{i}^{*}}+\frac{X_{i}^{*}}{\left(1+S_{i}^{*}\right)^{2}}\right)\left(\frac{1}{\tau_{i}^{*}} \cdot \frac{X_{i-1}^{*}}{X_{i}^{*}}\right)+\frac{S_{i}^{*}}{1+S_{i}^{*}} \cdot \frac{X_{i}^{*}}{\left(1+S_{i}^{*}\right)^{2}}, \\
\operatorname{trace} J=-\frac{1}{\tau_{i}^{*}}-\frac{X_{i}^{*}}{\left(1+S_{i}^{*}\right)^{2}}-\frac{X_{i-1}^{*}}{X_{i}^{*}} \cdot \frac{1}{\tau_{i}^{*}} .
\end{gathered}
$$

The trace of the Jacobian is negative and the determinant of the Jacobian is positive for all physically meaningful solutions $\left(X_{i}^{*}>0\right.$ and $\left.S_{i}^{*}>0\right)$. Consequently, provided that washout does not occur in the first reactor, the steady-state solution of the cascade is locally stable.

Steady-state diagrams showing the dimensionless effluent concentration $\left(S_{n}^{*}\right)$ leaving an $n$-reactor cascade as a function of the dimensionless total residence time are shown in Figure 3 for $n=1,2, \ldots, 4$. In this figure only physically meaningful solutions have been plotted. That is, in Figures 3(a) the stable solution for sufficiently low residence times $\left(\tau_{t}^{*}<\tau_{t, \mathrm{tr}}^{*}\right)$ are the lines $S_{n}^{*}=S_{0}^{*}=40$. In this figure, it is assumed that the residence time in each reactor is equal and given by $\tau_{i}^{*}=\tau_{t}^{*} / n$.
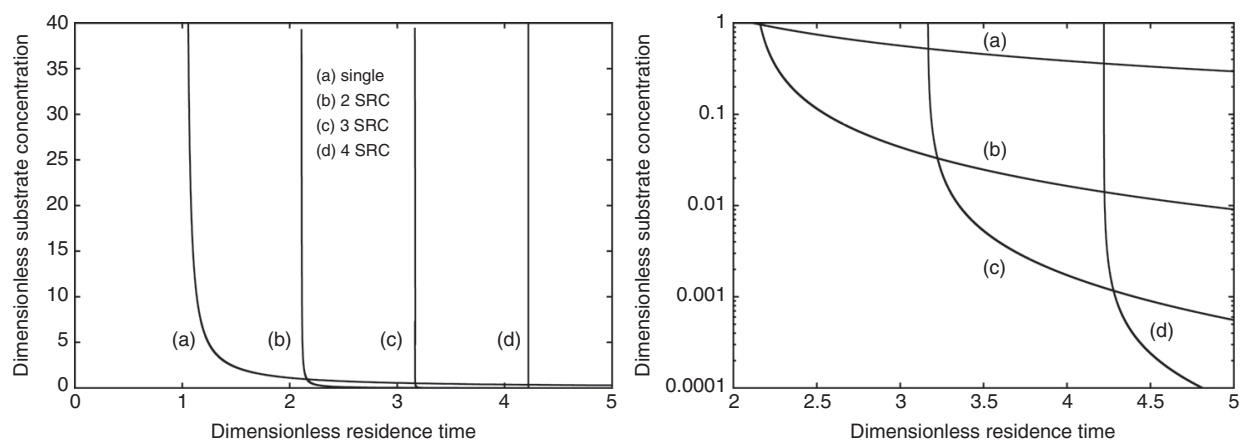

Figure 3: Steady-state diagrams showing the variation of dimensionless effluent concentration $\left(S_{n}^{*}\right)$ leaving a $n$ reactor standard reactor cascade (SRC) as a function of the total dimensionless residence time $\left(\tau_{t}^{*}\right)$ in the cascade. Parameter values: dimensionless death rate, $k_{d}^{*}=0.028$; dimensionless feed concentration, $S_{0}^{*}=40$. 
Figure 3(a) shows a very rapid decrease in the effluent concentration leaving the cascade as the residence time is increased through the transcritical bifurcation. Figure 3(b) shows that a total residence time $\tau_{t}^{*}=4$ the double reactor cascade delivers an order of magnitude decrease in the effluent concentration compared to that obtained in a single reactor, whilst the triple reactor cascade delivers a similar order of magnitude improvement compared to the double reactor cascade. At a residence time $\tau_{t}^{*}=5$ the effluent concentration leaving a four-reactor cascade is less than $0.00025 \%$ of the value in the influent. Figure 3(b) is a blow-up of Figure 3(a) showing in more detail the intersection of the steadystate solution curves. This illustrates the well-known result that, although large residences times are required in a single reactor to achieve a low effluent concentration, in a cascade low effluent concentrations are obtained with relatively small residence times. Furthermore, at relative low residence times we see that the effluent concentration leaving a cascade with 2,3, or 4 reactors is lower than the minimum effluent concentration that can be achieved in a single reactor $\left(S_{1}(\tau=\infty)=k_{d}^{*} /\left(1-k_{d}^{*}\right) \approx 0.0289\right)$.

As noted by Abu-Reesh [7] in a related context, the optimal number of reactors in the cascade depends upon the desired performance. For example, if the target is to remove $90 \%$ of the pollutant, corresponding to an effluent concentration leaving the final reactor $S_{n}^{*}=4$, than a single reactor minimises the total residence time. As the degree of pollutant removal increases, there are switching points when two reactors are better than one, three reactors better than two, four reactors better than three, etc.

As the number of reactors in the cascade increases, the washout time becomes larger than the residence time required in a smaller cascade to achieve a given effluent concentration. Thus, in practice, reactor cascades employing more than three reactors "are seldom employed" [14, chapter 12.2.2].

\section{Results for a step-feed reactor cascade (SFRC)}

In Section 4.1 we show that, provided that washout does not occur in the first reactor, the system has a unique steady-state solution and give the steady-state values of the substrate and cell-mass concentrations in the ith reactor of the cascade. In Section 4.2 the steady-state solution is shown to be locally stable. In these sections the residence times in teach reactor are arbitrary.
For the case when the residence time in each reactor is the same we establish in Section 4.1 that the steadystate solutions in the $i$ th reactor of the cascade are equal to those in the preceding reactor $\left(S_{i}^{*}=S_{i-1}^{*}, X_{i}^{*}=X_{i-1}^{*}\right)$.

\subsection{Steady-state solution branches}

The steady-state solutions in the $i$ th reactor of the cascade $(i>1)$ are found by solving the algebraic equations

$$
\begin{aligned}
& f\left(S_{i}^{*}, X_{i}^{*}\right)=\frac{(i-1)}{n \tau_{i}^{*}}\left(S_{i-1}^{*}-S_{i}^{*}\right)+\frac{S_{0}^{*}-S_{i}^{*}}{n \tau_{i}^{*}}-\frac{S_{i}^{*} X_{i}^{*}}{1+S_{i}^{*}}=0, \\
& g\left(S_{i}^{*}, X_{i}^{*}\right)=\frac{(i-1)}{n \tau_{i}^{*}}\left(X_{i-1}^{*}-X_{i}^{*}\right)-\frac{1}{n \tau_{i}^{*}} X_{i}^{*}+\frac{S_{i}^{*} X_{i}^{*}}{1+S_{i}^{*}}-k_{d}^{*} X_{i}^{*}=0 .
\end{aligned}
$$

The solution of these equations is given by

$$
\left(S_{i}^{*}, X_{i}^{*}\right)=\left(S_{i}^{*}, \frac{1}{n \tau_{i}^{*}}\left[(i-1) S_{i-1}^{*}+S_{0}^{*}-i S_{i}^{*}\right] \frac{1+S_{i}^{*}}{S_{i}^{*}}\right),
$$

where the cell mass concentration $S_{i}^{*}$ satisfies the quadratic equation

$$
G\left(S_{i}^{*}\right)=a_{i} S_{i}^{*^{2}}+b_{i} S_{i}^{*}+c_{i}=0 .
$$

with solution

$$
S_{i}^{*}=\frac{-b_{i} \pm \sqrt{b_{i}^{2}-4 a_{i} c_{i}}}{2 a_{i}} .
$$

The coefficients in these equations are

$$
\begin{aligned}
a_{i}= & i\left[\left(1-k_{d}^{*}\right) n \tau_{i}^{*}-i\right] \\
b_{i}= & \left(i+k_{d}^{*} n \tau_{i}^{*}\right)\left[(i-1) S_{i-1}^{*}+S_{0}^{*}-i\right] \\
& -n \tau_{i}^{*}\left[(i-1)\left(S_{i-1}^{*}+X_{i-1}^{*}\right)+S_{0}^{*}\right] \\
& c_{i}=\left(i+k_{d}^{*} n \tau_{i}^{*}\right)\left[(i-1) S_{i-1}^{*}+S_{0}^{*}\right] .
\end{aligned}
$$

By calculation we find that

$$
\begin{aligned}
& G\left(S_{i}^{*}=0\right)=\left(1+k_{d}^{*} n \tau^{*}\right)\left[(i-1) S_{i}^{*}+S_{0}^{*}\right]>0, \\
& G\left(S_{i}^{*}=\frac{1}{i}\left[(i-1) S_{i-1}^{*}+S_{0}^{*}\right]\right) \\
& \quad=-\frac{n \tau_{i}^{*} X_{i}^{*}}{i}\left[S_{i-1}^{*}+(i-1) S_{0}^{*}+i(i-2) S_{i-1}^{*}\right]<0 .
\end{aligned}
$$

(Negativity of the latter bound follows from the fact that $i \geq 2$, we already have the exact solution in the first reactor of the cascade).

Following the reasoning of Appendix A, it follows that the solution corresponding to the choice of the 
positive square root in eq. (22) is not physically meaningful as either the substrate concentration $\left(S_{i}^{*}\right)$ or the cell mass concentration $\left(X_{i}^{*}\right)$ are negative. However, the solution corresponding to the choice of the negative square root is physically meaningful; both the cell mass and substrate concentrations are positive. Thus, given that washout does not occur in the first reactor, the solution in each reactor in the cascade is unique. Furthermore the root of interest is in the region

$$
0<S_{i}^{*}<\frac{1}{i}\left[(i-1) S_{i-1}^{*}+S_{0}^{*}\right] .
$$

When $i=2$ eq. (21) is a special case of [14], which treats the more general situation of a multistream reactor cascade in which the flow rates of the feed stream into the reactors of a SFRC are not necessarily equal. Herbert [13] obtained steady-state expressions for an even more general two-reactor configuration, in which the flow rates of the feed stream and the feed composition into the reactors of a SFRC are not necessarily equal, though he assumed that the microorganisms were immortal $\left(k_{d}^{*}=0\right)$. He generalised his solution the case of $n$ reactors.

We now arrive at a most remarkable feature of the non-standard cascade model with Monod kinetic: the substrate and biomass concentrations in the ith reactor are equal to those in the first reactor! By substitution of the expressions $S_{i}^{*}=S_{i-1}^{*}=S_{1}^{*}$ and $X_{i}^{*}=X_{i-1}^{*}=X_{1}^{*}$ into eq. (21) it is readily shown (particularly using a symbolic manipulation package such as Maple) that

$$
S_{i}^{*}=S_{1}^{*},
$$

and hence

$$
X_{i}^{*}=X_{1}^{*} .
$$

We immediately deduce from this that for the case of Monod kinetics there is no point in running a step-fed reactor cascade without recycle as there is no difference in performance over having a single reactor. This result is readily confirmed numerically by directly solving the model equations for a reactor cascade containing two or three reactors.

A similar result has been obtained for the case of a step-feed reactor with recycle, where the recycle is distributed amongst the reactors in the cascade [14, section 13.2.3]. This result was obtained by numerical integration of the governing equations.

\subsection{Stability of the steady-state solutions}

The Jacobian for a non-standard $n$-bioreactor cascade is given by a matrix of the form (17) in which the blocks $B_{i}$ and $J_{i}$, each having size $2 \times 2$, are defined by

$$
\begin{gathered}
B_{i}=\left(\begin{array}{cc}
\frac{i-1}{n \tau_{i}^{*}} & 0, \\
0 & \frac{i-1}{n \tau_{i}^{*}}
\end{array}\right), \\
J_{i}=\left(\begin{array}{cc}
-\frac{1}{\tau_{i}^{*}}-\frac{X_{i}^{*}}{\left(1+S_{i}^{*}\right)^{2}} & -\frac{S_{i}^{*}}{1+S_{i}^{*}} \\
\frac{X_{i}^{*}}{\left(1+S_{i}^{*}\right)^{2}} & \frac{-(i-1)}{\tau_{i}^{*}} \cdot \frac{X_{i-1}^{*}}{X_{i}^{*}}
\end{array}\right) .
\end{gathered}
$$

The analysis of this Jacobian matrix follows that in Section 3.2. The block $J_{1}$ has two eigenvalues with negative real parts when (Section 2.3)

$$
\begin{gathered}
\tau_{1}^{*} \geq \frac{1+S_{0}^{*}}{S_{0}^{*}-\left(1+S_{0}^{*}\right) k_{d}^{*}}, \\
0 \leq k_{d}^{*}<\frac{S_{0}^{*}}{1+S_{0}^{*}}
\end{gathered}
$$

It follows by a similar calculation to that performed in Section 3.2 that the block $J_{i}$ has eigenvalues with negative real parts for all physically meaningful solutions. Consequently, provided that washout does not occur in the first reactor, the steady-state solution of the nonstandard reactor cascade is locally stable.

In Section 4.1 we showed that the effluent concentration leaving a SFRC is the same as the effluent concentration leaving the first reactor of the SFRC. This is given in eq. (12). The steady-state diagram for a SFRC containing $n$ reactors is therefore given by line (a) in Figure 3 with the understanding that the label on the $x$-axis be replaced by nominal residence time in the SFRC.

\section{Discussion}

To analyse the equations for the non-standard reactor cascade, it was convenient to introduce the concept of a "nominal residence time" in the cascade. In this section, we show that the "nominal residence time" is the average residence time experienced by particles passing through the SFRC. For the sake of illustration, consider a SFRC containing 3 reactors of equal volume $(V)$. The residence time in the ith reactor of such a cascade is

$$
\tau_{i}=\frac{3}{i} \cdot \frac{V}{F},
$$

where $F$ is the flow-rate of the feed-stream. Thus the total residence time within the cascade of a particle entering the ith reactor of the cascade from the feed bypass stream is 


$$
\begin{aligned}
& i=1 \quad\left(\frac{3}{1}+\frac{3}{2}+\frac{3}{3}\right) \frac{V}{F}=\frac{11}{2} \frac{V}{F} \\
& i=2 \quad\left(\frac{3}{2}+\frac{3}{3}\right) \frac{V}{F}=\frac{5}{2} \frac{V}{F} \\
& i=3 \quad=\frac{3}{3} \frac{V}{F}=\frac{V}{F}
\end{aligned}
$$

An equal proportion of the feed enters each reactor. Thus the average residence time within the cascade of a particle in the feed stream is

$$
\begin{aligned}
\tau_{\mathrm{av}} & =\frac{1}{3}\left(\frac{11}{2} \frac{V}{F}+\frac{5}{2} \frac{F}{V}+\frac{2 V}{2 F}\right), \\
& =3 \frac{V}{F} \\
& =3 \tau_{i}=\tau_{\text {nom }} .
\end{aligned}
$$

This calculation can be generalised to show that in a SFRC containing $n$ reactors

$$
\tau_{\mathrm{av}}=n \tau_{i}=\tau_{\mathrm{nom}} .
$$

This equation states that the average time spent by a particle in a step-feed reactor is equal to the average time that a particle spends in a standard reactor cascade.

We now show that our conclusions about a stepfeed reactor cascade for Monod kinetics hold for a more general specific rate law. Consider a SFRC subject to a general specific rate law $\mu(S, X)$. The model equations are

$$
\begin{aligned}
& \frac{d S_{i}}{d t}=\frac{(i-1)}{n \tau_{i}}\left(S_{i-1}-S_{i}\right)+\frac{1}{n \tau_{i}}\left(S_{0}-S_{i}\right)-\mu\left(X_{i}, S_{i}\right) X_{i} \cdot \frac{1}{\alpha}, \\
& \frac{d X_{i}}{d t}=\frac{(i-1)}{n \tau_{i}}\left(X_{i-1}-X_{i}\right)+\frac{1}{n \tau_{i}}\left(X_{0}-X_{i}\right)+\mu\left(S_{i}, X_{i}\right) X_{i}-k_{d} X_{i},
\end{aligned}
$$

where the model parameters are defined in Section 2.1.1.

We now define dimensionless variables $S_{i}^{*}=S_{i} / \bar{S}$, $X_{i}^{*}=X_{i} /(\alpha \bar{X})$ and $t^{*}=\bar{t} t$, where the parameters $\bar{S}, \bar{X}$ and $\bar{t}$ are determined by the particular choice of the specific growth rate function $\mu(X, S)$. The dimensionless equations for the $i$ th reactor of the SFRC are now

$$
\begin{aligned}
\frac{d S_{i}^{*}}{d t^{*}}= & \frac{(i-1)}{n \tau_{i}^{*}}\left(S_{i-1}^{*}-S_{i}^{*}\right)+\frac{1}{n \tau_{i}^{*}}\left(S_{0}^{*}-S_{i}^{*}\right)-\mu^{*}\left(S_{i}^{*}, X_{i}^{*}\right) X_{i}^{*} \\
\frac{d X_{i}^{*}}{d t^{*}}= & \frac{(i-1)}{n \tau_{i}^{*}}\left(X_{i-1}^{*}-X_{i}^{*}\right)+\frac{1}{n \tau_{i}^{*}}\left(X_{0}^{*}-X_{i}^{*}\right) \\
& +\mu^{*}\left(S_{i}^{*}, X_{i}^{*}\right) X_{i}^{*}-k_{d}^{*} X_{i}^{*}
\end{aligned}
$$

where the parameter groups are: the dimensionless decay rate $\left[k_{d}^{*}=k_{d} / \bar{t}\right]$ the dimensionless specific growth rate function, $\left[\mu^{*}\left(S_{i}^{*}, X_{i}^{*}\right)=\mu\left(\bar{S} S_{i}, \alpha \bar{S} X_{i}\right) / \bar{t}\right]$; and the dimensionless residence time $\left[\tau_{i}^{*}=\bar{t} \tau_{i} \mathrm{~A}\right]$.

It is now straightforward to show that if $\left(S_{1}^{*}, X_{1}^{*}\right)$ is a steady-state solution in the first reactor then $\left(S_{i}^{*}, X_{i}^{*}\right)=\left(S_{1}^{*}, X_{1}^{*}\right)$ is a steady-state solution in the $i$ th reactor of the SFRC.

\section{Conclusion}

We have investigated two reactor cascade models for the interaction between a microorganism and a ratecontrolling substrate. The biochemical model used the Monod expression for the specific growth rate and included a microorganism decay coefficient. The cascade models were the standard reactor cascade, shown in Figure 1, and a step-feed reactor cascade, shown in Figure 2. The main novelty in our work is our analysis of the step-feed reactor cascade. In both cases we showed that, provided washout does not occur in the first reactor of the cascade, the solution in the ith reactor of cascade is unique and locally stable. In both cases the steady-state solution is found by solving a quadratic equation for the substrate concentration, taking the negative square root sign. We showed that the solution in the ith reactor of the step-feed reactor cascade $\left(S_{i}^{*}, X_{i}^{*}\right)$ is equal to the solution in the first reactor of the cascade $\left(S_{1}^{*}, X_{1}^{*}\right)$. We generalised this result to show that if $\left(S_{1}^{*}, X_{1}^{*}\right)$ is a steady-state solution in the first reactor of a SFRC for a general specific growth rate law $\mu^{*}\left(S^{*}, X^{*}\right)$ then $\left(S_{i}^{*}, X_{i}^{*}\right)=\left(S_{1}^{*}, X_{1}^{*}\right)$ is a steady-state solution for the $i$ th reactor of the SFRC. Thus the step-feed reactor cascade does not improve the performance over that of a single reactor. This is surprising as the step-feed reactor cascade is common in the implementation of the activated sludge process [1, 2]. We conclude that the step-feed reactor cascade is not appealing as a viable alternative to a conventional reactor design.

Acknowledgments: During part of this work MIN was a Visiting Fellow in the School of Physical Environmental \& Mathematical Sciences (PEMS), UNSW@ADFA. He thanks the members of PEMS for their collegiality.

Research funding: Sultan Qaboos University (Grant/ Award Number: "IG/SCI/DOMS/08/04", "IG/SCI/DOMS/ 14/04”). 


\section{Appendix A}

\section{A.1 Steady-state analysis}

Here we show that the solution of eq. (16) in the second reactor of a cascade $(i=2)$ is only physically meaningful $\left(S_{2}^{*}>0\right.$ and $\left.X_{2}^{*}>0\right)$ when the negative square root sign is taken. In doing so the only property of $S_{1}^{*}$ and $X_{1}^{*}$ that we use is that they are positive.

The steady-state equations for the concentrations inside the third reactor of a cascade $(i=3)$ are identical to those of the second reactor in the cascade except that the all indices have increased by one. It immediately follows that the physically meaningful steady-state solution in the third reactor, and hence any reactor in the cascade, is given by the negative square root in eq. (16).

In Section A.1.1 we establish the desired result for the non-degenerate case with $a_{i} \neq 0$. In Section A.1.2 we show that the steady-state solution is physically meaningful in the degenerate case that $a_{i}=0$.

\section{A.1.1 Positivity of the steady-state solution $\left(a_{i} \neq 0\right)$}

Consider the function

$$
\begin{aligned}
G\left(S_{2}^{*}\right) & =a_{2} S_{2}^{* 2}+b_{2} S_{2}^{*}+c_{2}, \\
a_{2} & =\left(1-k_{d}^{*}\right) \tau_{2}^{*}-1 \\
b_{2} & =-a_{2} S_{1}^{*}-\tau_{2}^{*} X_{1}^{*}-\left(1+k_{d}^{*} \tau_{2}^{*}\right) \\
c_{2} & =\left(1+k_{d}^{*} \tau_{2}^{*}\right) S_{1}^{*} .
\end{aligned}
$$

The coefficient $c_{2}$ is strictly positive because $k_{d}^{*} \geq 0$, $S_{1}^{*}>0$ and $\tau_{2}^{*}>0$.

The roots of eq. (25) are given by

$$
S_{2}^{*}=\frac{-b_{2} \pm \sqrt{b_{2}^{2}-4 a_{2} c_{2}}}{2 a_{2}} .
$$

By calculation we have

$$
\begin{gathered}
G\left(S_{2}^{*}=0\right)=c_{2}>0, \\
G\left(S_{2}^{*}=S_{1}^{*}\right)=-\tau_{i}^{*} S_{1}^{*} X_{1}^{*}<0,
\end{gathered}
$$

as $S_{1}^{*}>0, X_{1}^{*}>0$ and $\tau_{1}^{*}>0$.

In the case when $a_{2}>0$ the calculations (27) and (28) lead to the conclusion that eq. (25) always has two solutions: one in the region $0<S_{2}^{*}<S_{1}^{*}$ and one in the region $S_{1}^{*}<S_{2}^{*}$. The solution with $S_{1}^{*}<S_{2}^{*}$ is not physically meaningful: eq. (14) shows that the corresponding concentration of microorganisms is negative. It follows that the solution of interest corresponds to the negative square root sign in eq. (26).
In the case when $a_{2}<0$ the calculations (27) and (28) lead to the conclusion that eq. (25) always has two solutions: a positive solution in the region $0<S_{2}^{*}<S_{1}^{*}$ and a negative solution $S_{2}^{*}<0$. The latter solution is not physically meaningful. It follows that the solution of interest corresponds to the negative square root sign in eq. (26).

\section{A.1.2 Positivity of the steady-state solution $\left(a_{i}=0\right)$}

In this section we show that the solution of eq. (16) is positive in the degenerate case when $a_{2}=0$. The case $a_{2}=0$ happens when $\tau_{2}^{*}=1+k_{d}^{*} \tau_{2}^{*}$. Now a straightfor-

\begin{tabular}{|c|c|c|}
\hline$F$ & $\begin{array}{l}\text { Flowrate through the } \\
\text { bioreactor. }\end{array}$ & $\left(\right.$ Lday $\left.^{-1}\right)$ \\
\hline$K_{s}$ & Monod constant. & $\left(\mathrm{mg} \mathrm{COD} \mathrm{L}^{-1}\right)$ \\
\hline$S_{j}$ & Substrate concentration. & $\left(\mathrm{mg} \mathrm{COD} \mathrm{L} \mathrm{L}^{-1}\right)$ \\
\hline$S_{j}^{*}$ & $\begin{array}{l}\text { Dimensionless substrate } \\
\text { concentration. } S_{j}^{*}=S_{j} / K_{s}\end{array}$ & $(-)$ \\
\hline$\hat{S}^{*}$ & $\begin{array}{l}\text { Dimensionless substrate } \\
\text { concentration along the no-washout } \\
\text { solution branch. }\end{array}$ & $(-)$ \\
\hline$S_{0}$ & Substrate concentration in the feed. & $\left(\mathrm{mg} \mathrm{COD} \mathrm{L}^{-1}\right)$ \\
\hline$S_{0}^{*}$ & $\begin{array}{l}\text { Dimensionless substrate } \\
\text { concentration in the feed. } \\
S_{0}^{*}=S_{0} / K_{s}\end{array}$ & $(-)$ \\
\hline SFRC & Step-feed reactor cascade. & \\
\hline $\mathrm{SRC}$ & Standard reactor cascade. & \\
\hline$V_{j}$ & Volume of a bioreactor. & $(\mathrm{L})$ \\
\hline$x_{j}$ & Concentration of microorganisms. & $\left(\mathrm{mg} \mathrm{MLSSL}^{-1}\right)$ \\
\hline$X_{j}^{*}$ & $\begin{array}{l}\text { Dimensionless microorganism } \\
\text { concentration. } X_{j}^{*}=X_{j} /\left(\alpha K_{s}\right)\end{array}$ & $(-)$ \\
\hline$x_{0}$ & $\begin{array}{l}\text { Concentration of microorganisms in } \\
\text { the feed. }\end{array}$ & $\left(\mathrm{mg} \mathrm{MLSSL}{ }^{-1}\right)$ \\
\hline$X_{0}^{*}$ & $\begin{array}{l}\text { Dimensionless microorganism } \\
\text { concentration in the feed. } \\
X_{0}^{*}=X_{0} /\left(\alpha K_{s}\right)\end{array}$ & $(-)$ \\
\hline$k_{d}$ & Death coefficient. & $\left(\right.$ day $\left.^{-1}\right)$ \\
\hline$k_{d}^{*}$ & $\begin{array}{l}\text { Dimensionless death coefficient. } \\
k_{d}^{*}=k_{d} / \mu_{m}\end{array}$ & $(-)$ \\
\hline $\mathrm{n}$ & $\begin{array}{l}\text { The number of reactors in a SRC or a } \\
\text { SFRC. }\end{array}$ & \\
\hline $\mathrm{t}$ & Time. & $\left(\right.$ day $\left.^{-1}\right)$ \\
\hline & Dimensionless time. $t^{*}=\mu_{m} t$ & $(-)$ \\
\hline
\end{tabular}
ward calculation shows that

$$
S_{2}^{*}=\frac{S_{1}^{*}}{1+X_{1}^{*}}<S_{1}^{*} .
$$

\section{Appendix B}

\section{B.1 Symbols used}

A subject $j$ refers to a property of the $j$ th reactor in a reactor cascade containing $n$ reactors. 
$\alpha \quad$ Yield factor.

$\mu \quad$ Specific growth rate model.

$\mu_{m} \quad$ Maximum specific growth rate.

$\tau \quad$ Residence time.

$\tau_{\mathrm{av}} \quad$ Average residence time in a SFRC.

$$
\tau_{\mathrm{av}}=\tau_{\text {nom }} \text {. }
$$
$\tau_{\text {nom }} \quad$ Nominal residence time through a
SFRC with reactors of equal size.

$$
\tau_{\text {nom }}=n \tau_{i}
$$

$\tau^{*} \quad$ Dimensionless residence time.

$$
\tau^{*}=V \mu_{m} / F
$$

$\tau_{\mathrm{tr}}^{*} \quad$ The value of the dimensionless residence time at the transcritical bifurcation.

\section{References}

1. Grady Jr C, Daigger G, Lim H. Biological wastewater treatment, 2nd ed., Chapter 7. Boca Raton, FL: CRC Press, 1999a:231-94.

2. Henze M, Grady Jr C, Gujer W, Marais G, Matsuo T. A general model for single-sludge wastewater treatment systems. Water Res 1987;21:505-15.

3. Monod J. The growth of bacterial culture. Annu Rev Microbiol 1949;3:371-94.

4. Nelson M, Kerr T, Chen X. A fundamental analysis of continuous flow bioreactor and membrane reactor models with death and maintenance included. Asia Pac J Chem Eng 2008;3:70-80. dx. doi.org/10.1002/apj.106

5. Grady Jr C, Daigger GLim H. Biological wastewater treatment, Chapter 12. New York and Basel: Marcel Dekker, Inc., 1980a:365-432.
6. Grady Jr C, Daigger G, Lim H. Biological wastewater treatment, 2nd ed. Boca Raton, FL: CRC Press, 1999b.

7. Abu-Reesh I. Optimal design of multi-stage bioreactors for degradation of phenolic industrial wastewater: theoretical analysis. J Biochem Technol 2010;2:175-81.

8. Dramé A, Harmand J, Rapaport A, Lobry C. Multiple steady state profiles in interconnected biological systems. Math Comput Model Interconnected Biol Syst 2006;12: 379-93.

9. Alqahtani R, Nelson M, Worthy A. A fundamental analysis of continuous flow bioreactor models with recycle around each reactor governed by Contois kinetics. III. Two and three reactor cascades. Chem Eng J 2012;183:422-32. dx.doi.org/10.1016/ j.cej.2011.12.061

10. Alqahtani R, Nelson M, Worthy A. A fundamental analysis of continuous flow bioreactor models governed by Contois kinetics. IV. Recycle around the whole reactor cascade. Chem Eng J 2013;218:99-107.

11. Carlsoon B, Zambrano J. Analysis of simple bioreactor modelsa comparison between Monod and Contois kinetics in IWA Special International Conference: Activated Sludge-100 years and Counting, 2014.

12. Yoon S-H. Important operational parameters of Membrane Bioreactor-Sludge Disintegration (MBR-SD) system for zero excess sludge production. Water Res 2003;37:1921-31.

13. Herbert D. Multi-stage continuous culture. In: Malek I, editor. Continuous culture of microorganisms, proceedings of the 2nd international symposium on continuous culture. San Diego, CA: Academic Press, 1964:23-44.

14. Grady Jr C, Lim H. Biological wastewater treatment. New York and Basel: Marcel Dekker, Inc, 1980b. 